\title{
Nanoscale Friction Behavior of the Ni-Film/Substrate System Under Scratching Using MD Simulation
}

\author{
X. M. Liu ·Z. L. Liu $\cdot$ Y. G. Wei
}

Received: 16 November 2011 / Accepted: 20 February 2012/Published online: 14 March 2012

(C) Springer Science+Business Media, LLC 2012

\begin{abstract}
The friction behavior of the nanoscratching process is investigated using molecular dynamic simulations by considering a sphere indenter sliding against a nickel nanofilm structure. In the film/substrate system, the interface-dominated friction process is studied during the nanoscratch process. The results indicate that the interface accommodates deformation during the scratch by absorbing plastic deformation (such as stacking faults and partial dislocations) and by allowing locally interface slip. The observed local material shuffling beneath the tip that was strongly affected by the interface and friction mechanisms, including material ploughing along the track, filling in of the track, and piling up of the chip in front of the tip, are discussed. The combination effects of both scratching depths and film thicknesses were also investigated.
\end{abstract}

Keywords Nanotribology · Friction-reducing ·

Friction mechanisms $\cdot$ Films $\cdot$ Dynamic modeling

\section{Introduction}

Friction problems are nearly inevitable in engineering and technological applications, and they play an important role in influencing the work efficiency [1]. For applications in the area of micro-engineering, such as microelectro-mechanical

X. M. Liu ( $₫)$ · Y. G. Wei

LNM, Institute of Mechanics, Chinese Academy of Sciences,

Beijing 100190, China

e-mail: xiaomingliu@lnm.imech.ac.cn

Z. L. Liu

Department of Mechanical Engineering, Northwestern

University, Evanston, IL 60208, USA systems (MEMS) and ultra-high-density recording, friction contact always takes place within the micro- or nano-size region [2]. On such a small scale, nanotribology resulting in adhesion, asperity contact, friction, and wear increases significantly. Thus, a full understanding of the friction law at the micro- or nano-scale is essential for designing small-scale devices. In past decades, considerable research has been focused on investigating the friction behaviors at the microor nano-scales based on theory and simulations. The concept of friction has been extended in a number of associated areas, such as using surface roughness [3, 4], phonon and electron excitation [5-7], and dislocation interaction theories [8-10].

For almost four decades lubrication has been employed in the friction system to reduce wear [11], with the lubricant used between surfaces to carry the load. For contact at the nanoscale, lubricant is inadequate since it may cause additional electrical forces or chemical reactions. In most such applications, a thin metal-coating layer is often introduced to the small-scale devices to enhance physical properties [12], and the specific case of nano-size coating layer has attracted much research interest [13, 14]. It is crucial to understand the interface effect on friction and wear properties at the nanoscale due to its importance in engineering applications. From experimental studies, layer thickness has been shown to play a key role in the mechanical properties of the multi-layer material [15], and a deformation mechanism transition exists from lattice dislocation to grain boundary motion at the critical layer thickness [16]. A considerable number of simulation studies have been recently carried out to address the interface effects. Cho et al. [10] investigated the interaction of dislocation with grain boundary for the nanoindentation case using a molecular dynamic (MD) simulation, and their results show that lower indentation resistance can be attributed to the dislocation interaction with the grain 
boundary. Saraev et al. [17] studied the resistance of a multi-layered metal thin film for the nanoindentation case and were able to demonstrate the Hall-Petch effect of layer thicknesses. However, these studies mostly focus on the interface effect for the nanoindentation cases, and few researchers have focused on the interface effect for nanoscratching friction case. Fang et al. [9] studied the friction response during the scratching of multi-layered films and observed both stick-slip and work-hardening behaviors during the scratching process. Cheng et al. [18] performed the scratching test on the Cu/Nickel (Ni) multilayer crystal system and observed that there is a misfit dislocation network which causes the frictional coefficient to be decreased. The material internal friction (plastic deformation) effect on tribological properties is not yet comprehensively studied, and the effect of how the film/ substrate interface affects the local plastic deformation evolution beneath the tip is still rather vague. During scratching, the interface induces local plastic deformation as well as microstructure evolution due to complex interactions between dislocations, stacking faults and interfaces. These changes should be systematically studied in terms of friction behavior. In the study reported here, we investigate the nanoscale friction behavior of a Ni-film/substrate system (one layer of film on the substrate) under conditions of nanoscratching on film by using MD simulations. The emphasis of the research was on studying how the internal friction at the grain boundary (GB) affects the tribological properties of the system. Ni was chosen because it is widely used in many industries, especially in surfacecoating technology. Both the effects of scratch depth and film thickness on the nano-friction behavior were studied. Detailed micro-plastic behavior around the indenter is discussed, including material ploughing, piling up, and dislocation generation and propagation, among others.

\section{Simulation Model}

In the present study, MD simulation is used to describe the nanoscratch process of a bicrystal Ni model containing $\Sigma 5$ twist grain boundaries. The embedded-atom method (EAM) potential [19] for $\mathrm{Ni}$ is adopted. A spherical indenter with radius $R$ exerts a force of magnitude by Eq. 1 on the atoms at the (010) surface [20]:

$P=-k\left(R-r_{I}\right)^{2}$ for $\quad r_{I}<R$

where $k$ is the effective stiffness of the indenter, $R$ is radius of the spherical indenter and $r_{I}$ is the distance from the center of the spherical indenter to the center of the atom $I$. The force is repulsive, and $P(r)=0$ for all the atoms which have $r_{\mathrm{I}}>R$. The radius $R$ is taken as $20 \AA$ in the present simulations. As shown in Fig. 1, a one-layer film

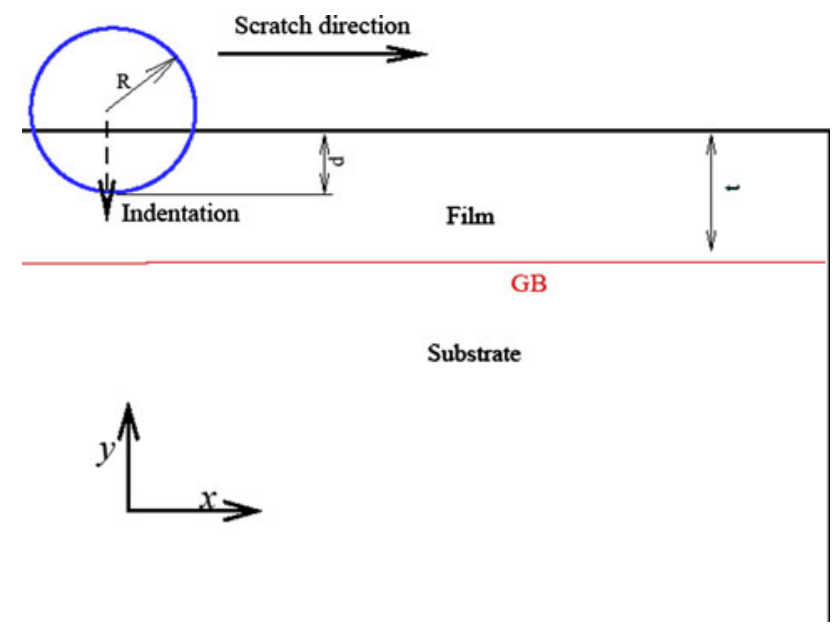

Fig. 1 Sketch of present scratch model. $G B$ Grain boundary

with thickness $t$ is on the top of the surface, with the crystal orientation of $x<-30-4>, y<010>, z<40-3>$; the substrate has the crystal orientation of $x<1 \quad 0 \quad 0>, y<01$ $0>, z<001>$. The interface between the film and substrate is a $\Sigma 5$ coincident twist grain boundary with a misorientation angle of $36.87^{\circ}$. The normal direction of the interface is $y<0 \quad 10>$. The simulated model consists of approximately 2,000,000 atoms and has a dimension of $x \times y \times z=563.2 \times 150.8 \times 281.6 \AA$. It is carefully selected so that the box has periodic boundaries in the $x$ and $z$ directions for both the film and substrate. Four layers of atoms are fixed in the $x$ and $y$ directions, and the scratch is conducted on the (010) surface in the $x<-3 \quad 0 \quad-4>$ direction.

MD simulations reported in this work are performed using large-scale atomic/molecular massively parallel simulator (LAMMPS) developed by Plimpton and coworkers [21]. The initial film structure is relaxed to the minimum energy configuration by using the conjugate gradient method, and the relaxed structure is taken as the initial configuration for scratching simulation. The entire process of the scratching simulation includes three steps: indentation, relaxation and scratch. Normal force and shear (friction) force are defined as the summing resultant forces at the indenter tip and are calculated during the scratching process. To keep the computational time into a reasonable value, the scratching speed is chosen to be $100 \mathrm{~m} / \mathrm{s}$ in the current MD simulation, which is a typical value in usual nano-scratching simulations $[22,23]$. To reduce the influence of thermal fluctuation on nucleation and the propagation of defects, we adopt the canonical ensemble via an external Nosé-Hoover thermostat [24] at a temperature of $1 \mathrm{~K}$. For comparison purposes, we also performed some simulations with a lower scratch velocity and higher temperature. In order to investigate the influence of the interface between film and substrate on the nano-friction 
phenomenon, the relationships between indenting depth on the film at various values of $\boldsymbol{d}$ and different film thickness $\boldsymbol{t}$ are studied as shown in Fig. 1. Friction behavior for Ni monolithic material under scratching and the same condition (scratch depth and move velocity) is also simulated for comparison purposes.

\section{Result and Discussion}

\subsection{Softening and Ultra-Low Friction at Shallow Scratch $\boldsymbol{d}=5 \AA$}

The relationship of normal force with scratch displacement at a scratch depth of $\boldsymbol{d}=8 \AA$ is shown in Fig. 2c. It can be seen that the normal force curves have similar initial slopes in the indentation processes and that the film thickness does not affect the elastic response [25], but does affect the yielding behavior. In the film with thickness $t=10 \AA$, the first displacement burst point appears around indentation depth $\boldsymbol{d}_{\text {cri }}=2.5 \AA$. The burst value increases with increasing film thickness, $\boldsymbol{d}_{c r i}=4 \AA$ for $\boldsymbol{t}=20 \AA$ and $\boldsymbol{d}_{\text {cri }}=5 \AA$ for $\boldsymbol{t}=40 \AA$, which is nearly the same as that in the bulk case. The subsequent response after first yielding shows that the interface causes the reduction of the plastic response, which is similar to findings reported earlier [17, 26]. Nix and coworkers [27] also indicated that the cause of such softening during the indentation process is due to dislocation emission from the interface or dislocation absorption at the interface. As a consequence, at the scratch depth of $\boldsymbol{d}=5 \AA$, normal force decreases with decreasing film thickness, as shown in Fig. 2a. The friction force is characterized in a different way, as shown in Fig. $2 b$, and its value is very small for a thicker film $t=20$ and $40 \AA$. However, the friction force changes abruptly to a big amplitude for the thinner film case $(t=10 \AA)$. In order to study the dynamic friction behavior of the system, the average forces over the scratch displacement $s$ (from $s=50$ to $s=280 \AA$ ) are calculated, as shown in Fig. 3. It can be seen from Fig. $3 \mathrm{a}$ that the shallow scratch $(\boldsymbol{d}=5 \AA)$ at film thickness $t=10 \AA$ corresponds to a large friction but that there is a perfect lubrication property at film thicknesses $t=20$ and $40 \AA$, as well as the bulk while $\boldsymbol{d}=5 \AA$ A The friction coefficient at scratch depth $\boldsymbol{d}=5 \AA$ also exhibits the same trend, as shown in Fig. 4 .

In order to study why the ultra-low friction behavior is obtained and why the friction coefficient changes abruptly with decreasing film thickness at the scratch depth $\boldsymbol{d}=5 \AA$, we analyzed the displacement field of the film/ substrate system under scratch; the $X-Y$ side views of the displacement field after the scratch are presented in Fig. 5. At the scratch depth $\boldsymbol{d}=5 \AA$, a large area of plastic deformation along the scratch track appears in the film with
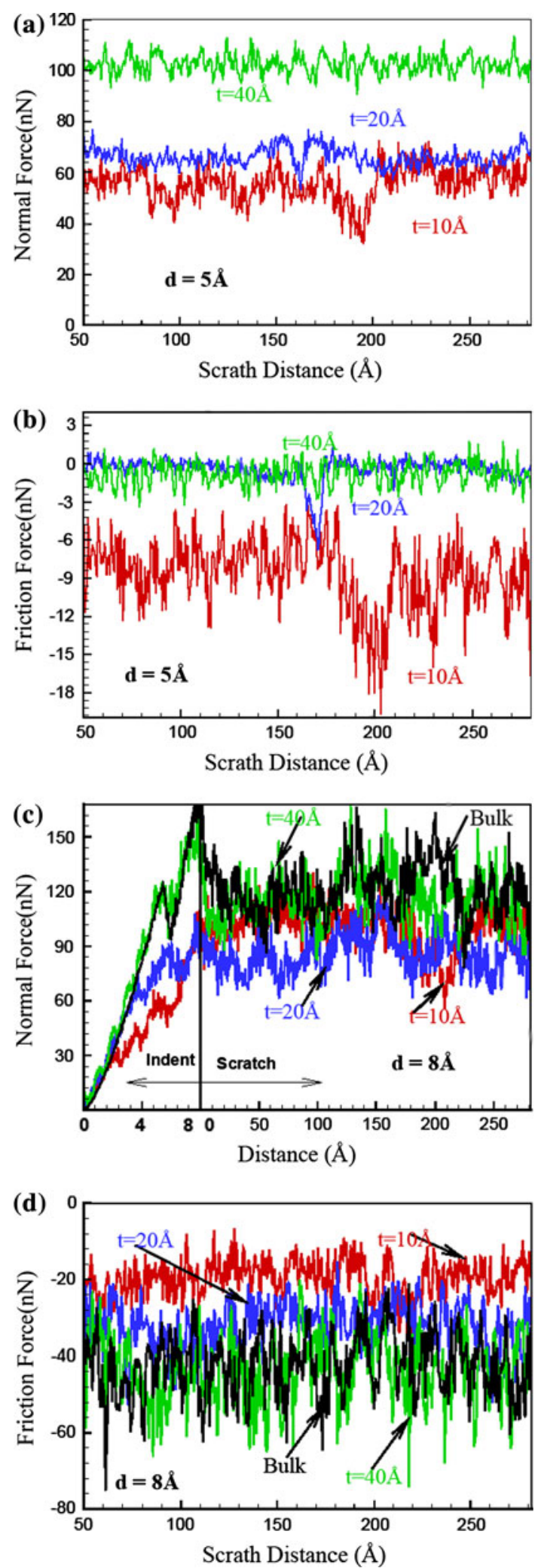

Fig. 2 Typical normal forces and cut forces as a function of the scratch distance $(\boldsymbol{d})$ associated with different film thicknesses: a normal forces $\boldsymbol{d}=5 \AA$, b friction forces $\boldsymbol{d}=5 \AA$, c normal forces $\boldsymbol{d}=8 \AA$, d friction forces $\boldsymbol{d}=8 \AA$. Film thicknesses $(\boldsymbol{t})=10,20$, $40 \AA$ 

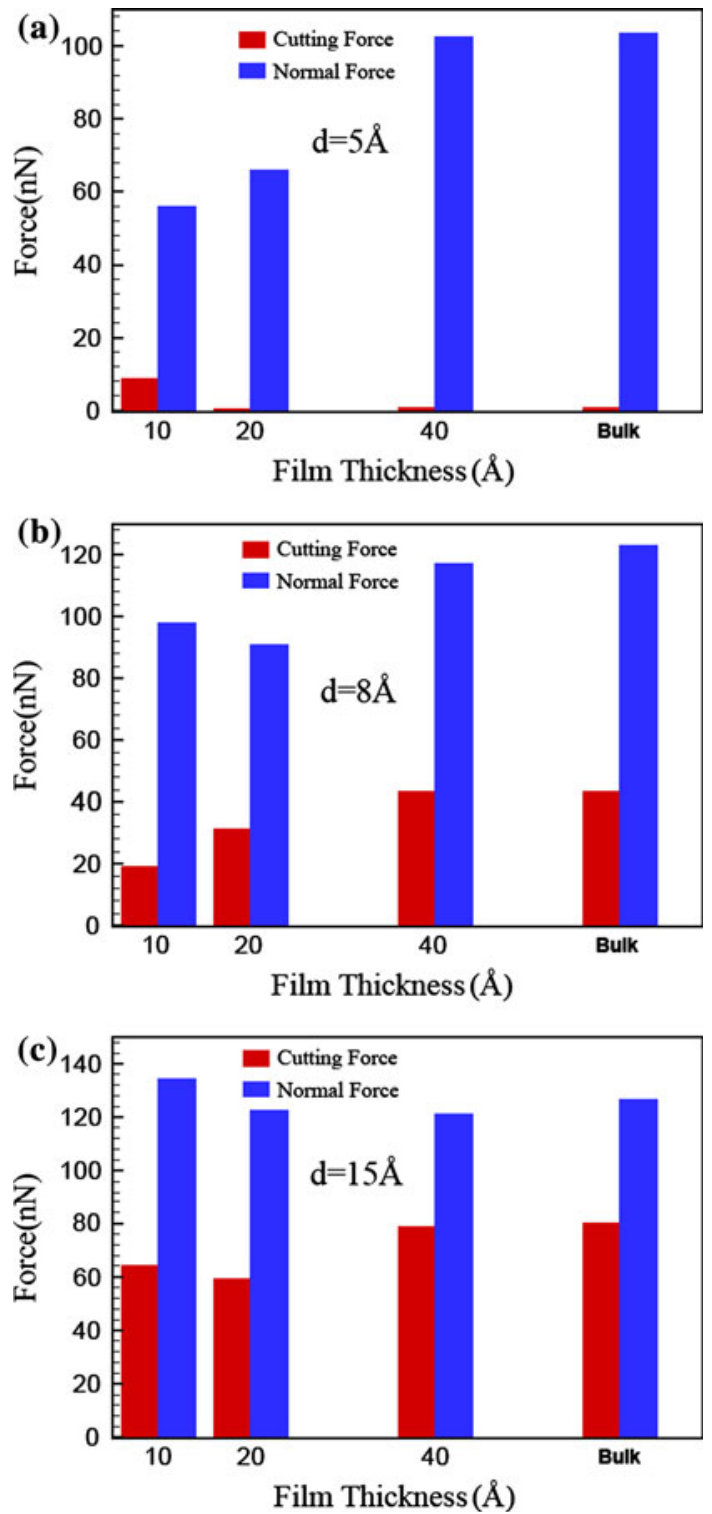

Fig. 3 Average forces as a function of the scratch distance associated with different indentation depths: a $\boldsymbol{d}=5 \AA, \mathbf{b} \boldsymbol{d}=8 \AA$, $\mathbf{c} \boldsymbol{d}=15 \AA$

thickness $t=10 \AA$, as shown in Fig. 5a, while almost no plastic deformation occurs in the cases of $t=20$ and $40 \AA$, as shown in Fig. 5 b, c. The deformation below the tip recovers during the relaxation stage after the tip moves forward. This is why the ultra-low friction occurs in thicker film cases. It should be noted that in Fig. 5a, dislocation absorption and stacking fault arresting are the main operating mechanisms within the thinner film, and the amorphous structure which is triggered by complex local reaction at interface is attributed to the increase in friction force. This can be proved by observing the atomistic defects around the tip for several film thicknesses at the beginning of scratch with the indentation depth $\boldsymbol{d}=5 \AA$, as shown in Fig. 6. Amorphous structures are formed in the

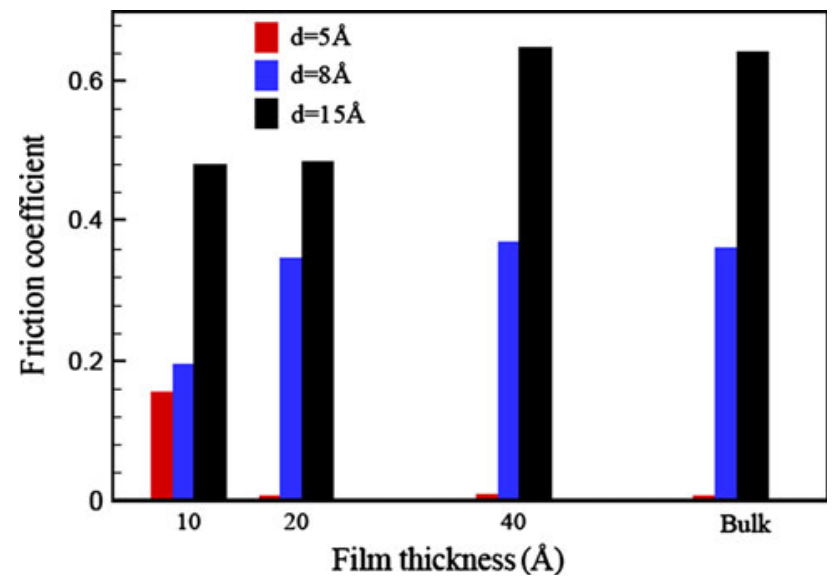

Fig. 4 Friction coefficient as function of film thickness and scratch depth

thinner film $(t=10 \AA)$ as the result of absorption of local plastic deformation at the interface, as shown in Fig. 6a. In the case of $t=20 \AA$, partial dislocations propagate from the subsurface to the interface, and the trans-film stacking faults are formed, as shown in red in Fig. 6b. However, the stacking faults are easily recovered after the indenter moves forward, indicating that the plastic deformation caused in the indentation process is healed during retraction. In the case of $\boldsymbol{t}=40 \AA$, stacking faults extend to the subsurface below the tip, as shown in Fig. 6c; however, the plastic deformation is also recovered in the scratch process after the indenter tip moves forward, as shown by the quick recovery of the surface. This phenomenon has also been observed in recycle indentation simulations [20]. These results clearly show that surface re-healing is a very effective way to recover surface plastic deformation at the nano-scale. Thus, it can be concluded that the amorphous structure which is induced by local interface slip in the thinner film hinders the recovery of deformation after the indenter tip moves forward. This phenomenon also explains why the ultra-low friction does not hold in the thinner film, and it can be also indicated by the average displacement profile, as shown in Fig. 7a. Atoms below the tip show a very small displacement in the cases of $t=20$ and $40 \AA$ because most of the deformation is recovered by rehealing. To the contrary, large movement appears in the film with $\boldsymbol{t}=10 \AA$ because both dislocations and stacking faults are absorbed by the interface. Thus, permanent deformation is produced, and the surface deformation can not be recovered by the high-surface mobility of atoms. The subsurface plastic reaction induced by the interface is the cause of the onset of wear.

In the experiment by Gosvami et al. [28], the atomically flat metal surface was found to exhibit very low frictional dissipation over a range of several nanonewtons, and there was almost no surface damage observed during low-load 


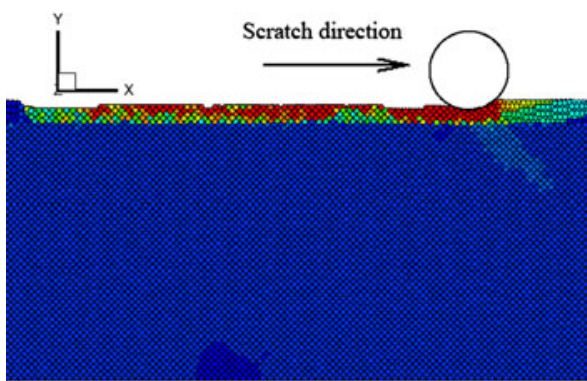

(a)

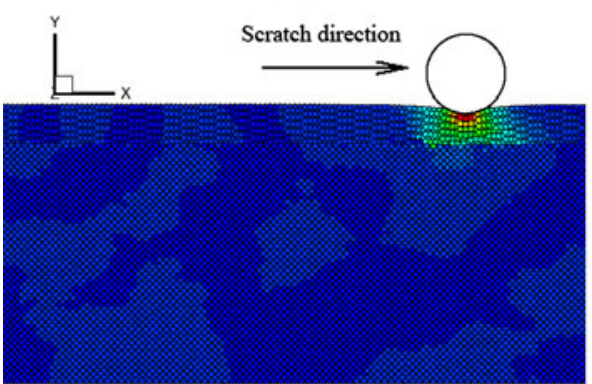

(b)

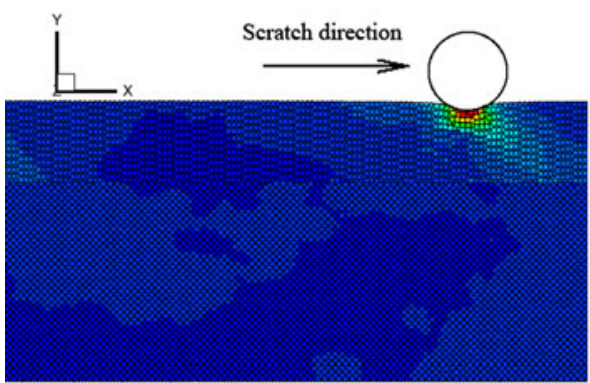

(c)

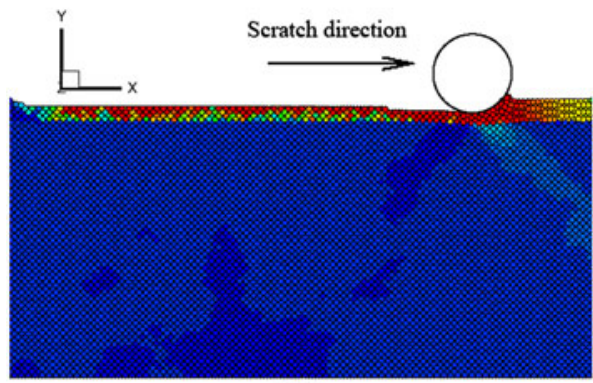

(d)

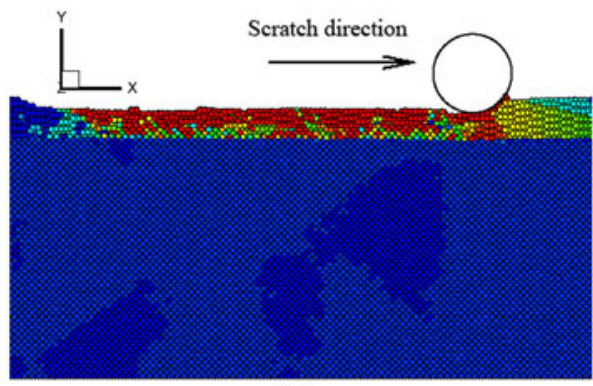

(e)

Fig. 5 Displacement field after scratching the system at a distance of $280 \AA$ with different film thicknesses: a $\boldsymbol{d}=5 \AA, \boldsymbol{t}=10 \AA$; b $\boldsymbol{d}=5 \AA, \boldsymbol{t}=20 \AA$; $\mathbf{c} \boldsymbol{d}=5 \AA, \boldsymbol{t}=40 \AA$; $\mathbf{d} \boldsymbol{d}=8 \AA, \boldsymbol{t}=10 \AA$

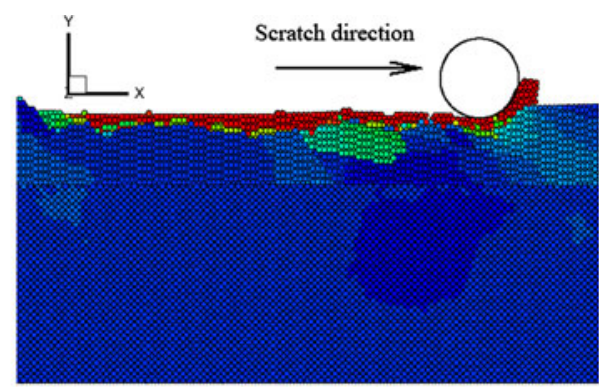

(f)

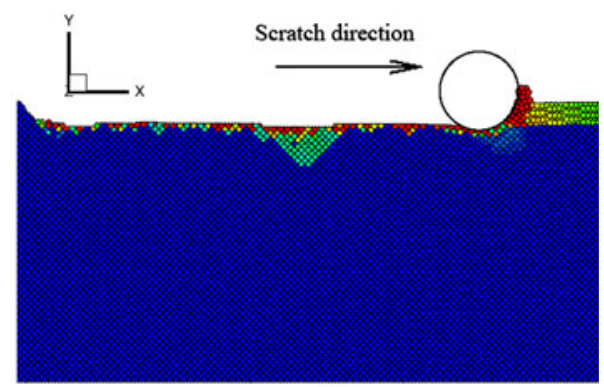

(g)

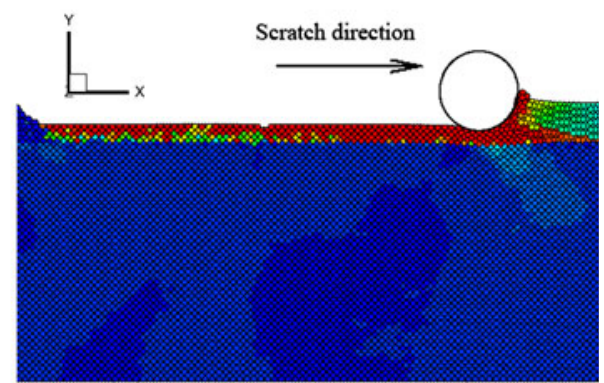

(h)

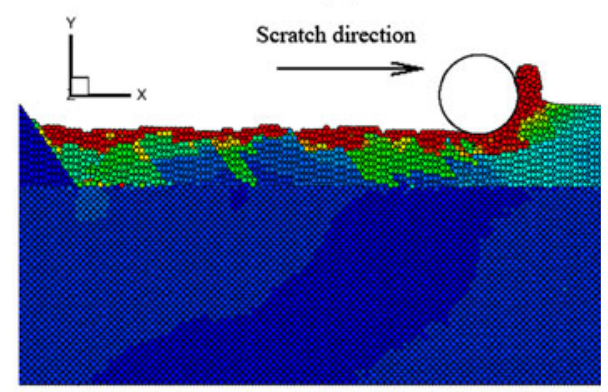

(i) e $\boldsymbol{d}=8 \AA, \boldsymbol{t}=20 \AA$; $\mathbf{f} \boldsymbol{d}=8 \AA, \boldsymbol{t}=40 \AA$; $\mathbf{g} \boldsymbol{d}=15 \AA, \boldsymbol{t}=10 \AA$; h $\boldsymbol{d}=15 \AA, \boldsymbol{t}=20 \AA$; I $\boldsymbol{d}=15 \AA, \boldsymbol{t}=40 \AA$ (all the figures are labeled in the same legend in a with the unit of $\AA$ ) 


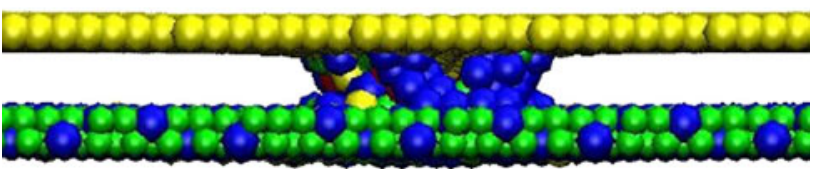

(a)

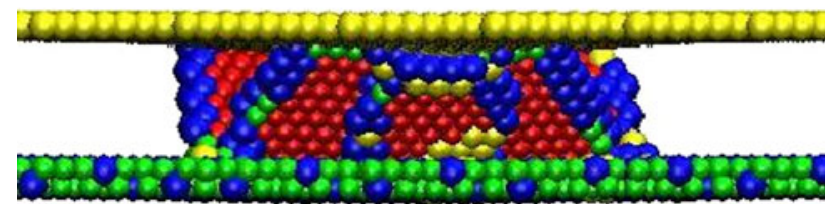

(b)

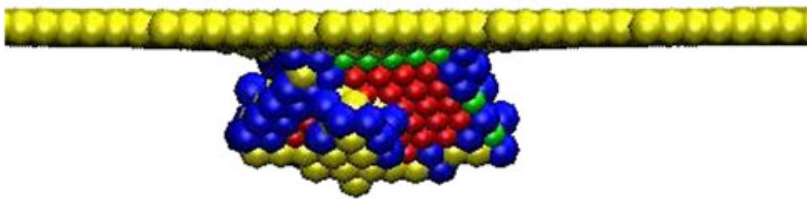

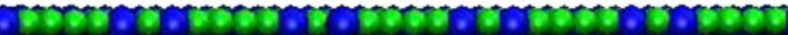

(c)

Fig. 6 Atomic structures beneath the indenter tip for various of film thickness $\boldsymbol{d}=5 \AA$ : $\mathbf{a} \boldsymbol{t}=10 \AA$, b $\boldsymbol{t}=20 \AA$, $\mathbf{c} \boldsymbol{t}=40 \AA$

scratch measurements. Our results suggest that the highsurface mobility of atoms can be taken as one of the reasons why the flat metal surface exhibits very low frictional dissipation-namely, it can restore the metal surfaces during the shallow scratch process. Plastic deformation can be healed if it does not react in a complex manner with the microstructure at the interface, and amorphous structure caused by severe deformation may induce the onset of wear (Table 1).

\subsection{Effects of Scratch Depth and Film Thickness}

The average normal and shear (cutting) forces during the scratch process are shown in Fig. 3. It can be seen that: (1) at a shallow scratch depth $\boldsymbol{d}=5 \AA$, normal force decreases with decreasing film thickness, and the softening phenomenon is caused by the interface; (2) at the scratch depth $\boldsymbol{d}=8 \AA$, normal force at a film thickness of $\boldsymbol{t}=10 \AA$ is a little larger than that of $t=20 \AA$ as the result of intensive dislocation and stacking fault interactions with the interface, and friction force decreases with decreasing film thickness; (3) At depth of $\boldsymbol{d}=15 \AA$, the deviation of the normal forces is small for all film thicknesses, and only a little hardening at $t=10 \AA$ case is observed. The friction forces have approximately the same value for film thickness $t=10$ and $20 \AA$ because in these two cases, the scratch depth $\boldsymbol{d}=15 \AA$ penetrates or approaches the interface, with the result that the interface may take on an
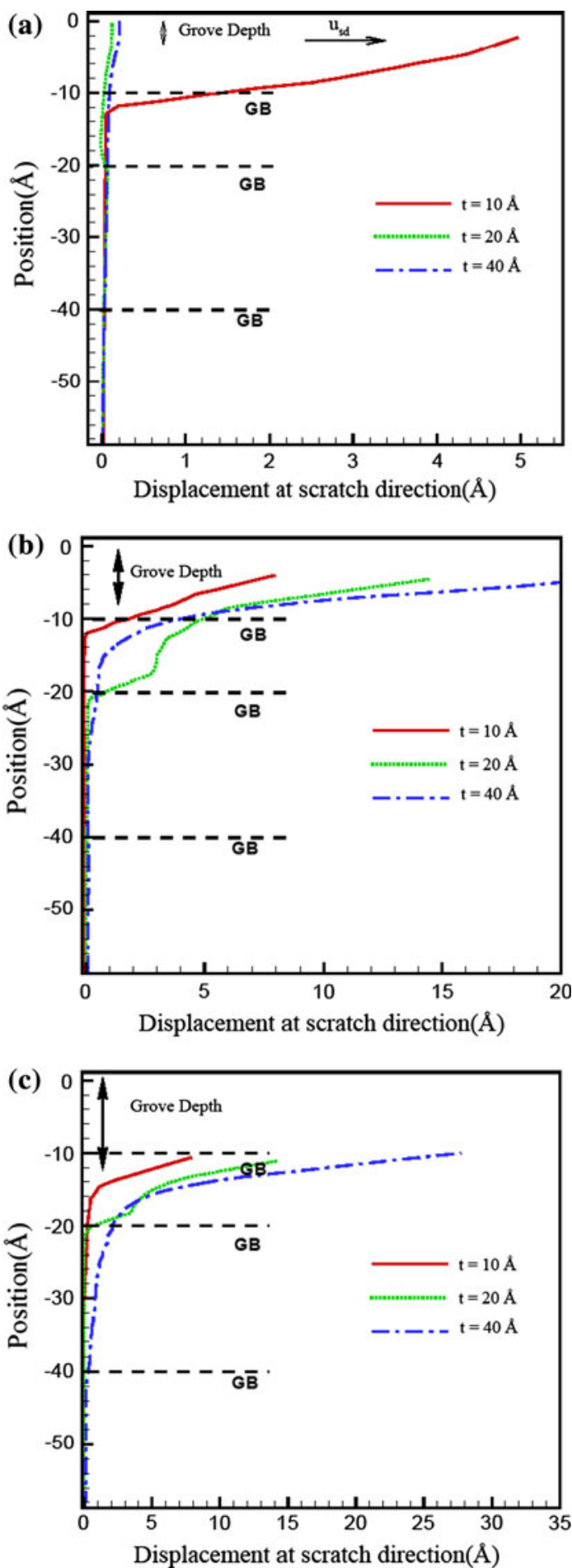

Fig. 7 Average atom's displacement profile at the scratch direction beneath the tip along the scratch track $\left(\boldsymbol{u}_{s d}\right.$ indicates the scratch direction): $\mathbf{a} \boldsymbol{d}=5 \AA, \mathbf{b} \boldsymbol{d}=8 \AA, \mathbf{c} \boldsymbol{d}=15 \AA$. We take an average of atom's displacement along the scratch track between 50 and $280 \AA$, neglecting the first $50 \AA$ distance in order to minimize the effect of the static-dynamic transition

effect similar to the friction force. The friction coefficient as the function of both film thickness and scratch depth is shown in Fig. 4: it decreases with decreases in both scratch 
Table 1 Friction map

\begin{tabular}{llll}
\hline $\begin{array}{l}\text { Film } \\
\text { thickness } \\
(t)\end{array}$ & Scratch depth $(\boldsymbol{d})$ & $8 \AA$ & $15 \AA$ \\
\cline { 2 - 3 } & $5 \AA$ & $\begin{array}{c}\text { Finite friction (amorphous structure, trans-film } \\
\text { stacking faults) }\end{array}$ & $\begin{array}{l}\text { Low friction (interface-mediated deformation, trans-film stacking faults, } \\
\text { interface slip) }\end{array}$ \\
$20 \AA$ & $\begin{array}{c}\text { Ultra-low friction (surface recovery due to } \\
\text { high-surface mobility of atoms) }\end{array}$ & $\begin{array}{c}\text { High friction (ploughing, piling up, } \\
\text { single slip V-dislocation nucleation) }\end{array}$ & $\begin{array}{c}\text { High friction (ploughing, piling up, } \\
\text { multi-slip V-dislocation nucleation) }\end{array}$ \\
$40 \AA$ & &
\end{tabular}

Microstructure characteristics are given in parenthesis

depth and film thickness except at the very fine scratch depth $\boldsymbol{d}=5 \AA$.

The deformation fields during the scratch are shown in Fig. 5 for the study of the detailed friction mechanism. It can be seen that the deformation localization is observed on the film without propagating to the bulk, especially for thinner film cases $t=10$ and $20 \AA$, as shown in Fig. $5 \mathrm{~d}-$ $\mathrm{g}$, h. The interface acts as a strong barrier for the deformation propagation. In order to quantify how much deformation the atoms are subject to below the tip and how this relates to the measured friction coefficient, the average displacement profiles at the scratch direction below the tip along the scratch track after scratch displacement of $280 \AA$ were calculated, as shown in Fig. 7 . It can be seen: (1): at scratch depth of $\boldsymbol{d}=5 \AA$ (Fig. 7a), only large displacement appears in the film of $t=10 \AA$, and transporting atoms are one of the reasons why large friction occurs; (2) at both depths of $\boldsymbol{d}=8$ and $15 \AA$ (Fig. $7 b, c)$, respectively, displacement profiles display the similar trend, jumping at the GB, indicating that atom's movement is localized in the film. The surface atoms in the case of $\boldsymbol{t}=40 \AA$ have the largest movement. The extreme distortion causes the largest friction dissipation, which can explain why thicker film has the larger friction coefficient, as shown in Fig. 4.

\subsection{Effects of Dislocation Evolution and Local Plasticity}

In the scratch process, dislocation nucleation and propagation are very important issues. Thus, the dislocation evolution during the nanoscratch process of the film/substrate structure was investigated by analyzing the local crystalline order of all the atoms in the system at certain scratch displacements. Figure 8 shows the snapshots of bottom views of the subsurface during the scratch process with scratch depths of $\boldsymbol{d}=8$ and $15 \AA$, respectively. The pictures are visualized by visual molecular dynamics (VMD), an open source molecular visualization program [29]. Firstly, at scratch depth $\boldsymbol{d}=8 \AA$ (Fig. 8a), there are inter-film stacking faults being generated during scratch in thinner film cases, $t=10$ and $20 \AA$, and their preferential slip directions are both in $<-10-1>$ and $<10-1>$, as indicated by the local coordinate system. This demonstrates that the interface reacts with the leading partial dislocation and, therefore, no full dislocation is nucleated. While in thick film $t=40 \AA$, groups of V-shape dislocation consisting of Shockley partial dislocations are constructed and then propagate in the slip direction $<-1 \quad 0 \quad-1>$. The resolved stress is small in the other slip direction $<10-1>$, so no dislocation propagation appears. Comparison of the two bottom figures in Fig. 8a shows that the dislocations which move out of the cell from the right side (left figure) enter into the box from the left side (right figure); the reason for this is that the periodic boundary condition is applied in the simulation model. It can be concluded that the thicker film prefers to form the $\mathrm{V}$-shape dislocation propagation below the surface rather than to form stacking fault intersecting the film. Secondly, at scratch depth $\boldsymbol{d}=15 \AA$ (Fig. 8b), there are inter-film stacking faults in thinner films of both $t=10$ and $20 \AA$, and these are similar to the ones in the previous case shown in Fig. 8a. However, the influence region increases since the deformation becomes larger with increasing $\boldsymbol{d}$. In contrast, in thick film $\boldsymbol{t}=40 \AA$, few Vshape dislocations are observed compared with the cases shown in Fig. 8a because (1) the deeper scratch depth corresponds to bigger $\mathrm{V}$-shape dislocations, thus the higher force is needed for activation of the bigger V-dislocations and (2) plastic deformation tends to localize below the tip at the deeper scratch depth, which can be seen by comparing left-down figures in both Fig. 8a, b, and the large stacking faults and local amorphous structures are formed at the deeper scratch. The stress concentration activates the Vdislocations in multiple slip systems, as indicated by arrows in Fig. 8b. Jiang and coworkers [10] also observed that with increasing indent depth, multiple dislocation nucleations are induced by the large stress in the substrate-and not individual events of dislocation nucleation. Our simulations show similar results. It can be concluded that the required friction forces for trans-film stacking fault $\boldsymbol{F}_{\text {stack }}$, single slip V-dislocation $\boldsymbol{F}_{\text {single }}$, and multi-slip V-dislocation $\boldsymbol{F}_{\text {multi }}$ have the following relationship: 
Fig. 8 Subsurface

microstructure after scratch distance of $60 \AA$ (left column) and $280 \AA$ (right column); the bottom views are shown in order to see the microstructure evolutions. Red circles V-shape dislocation moving forward in the direction illustrated by the arrow, open triangle indicates the position of the indenter. Scratch depth $\boldsymbol{d}=8 \AA$ (a) and $\boldsymbol{d}=15 \AA$ (b), from top to bottom, $t=10,20$, and $40 \AA$. Local crystalline order analysis was used to visualize defects in the system, The transparent atoms with local face-centered cubic (FCC) order represent the perfect FCC lattice; part of the atoms which have 12 nearest neighbors with local hexagonal close-packed (HCP) order are colored with red representing those in the stacking faults, blue representing the rest with 12 nearest neighbors in order to represent atoms in the dislocation core, green indicating atoms with 10 or 11 nearest neighbours representing those in the vacancies, and yellow indicating those with fewer than ten nearest neighbours and standing for fully disordered ones, which represent the atoms at the surfaces (Color figure online) (a)
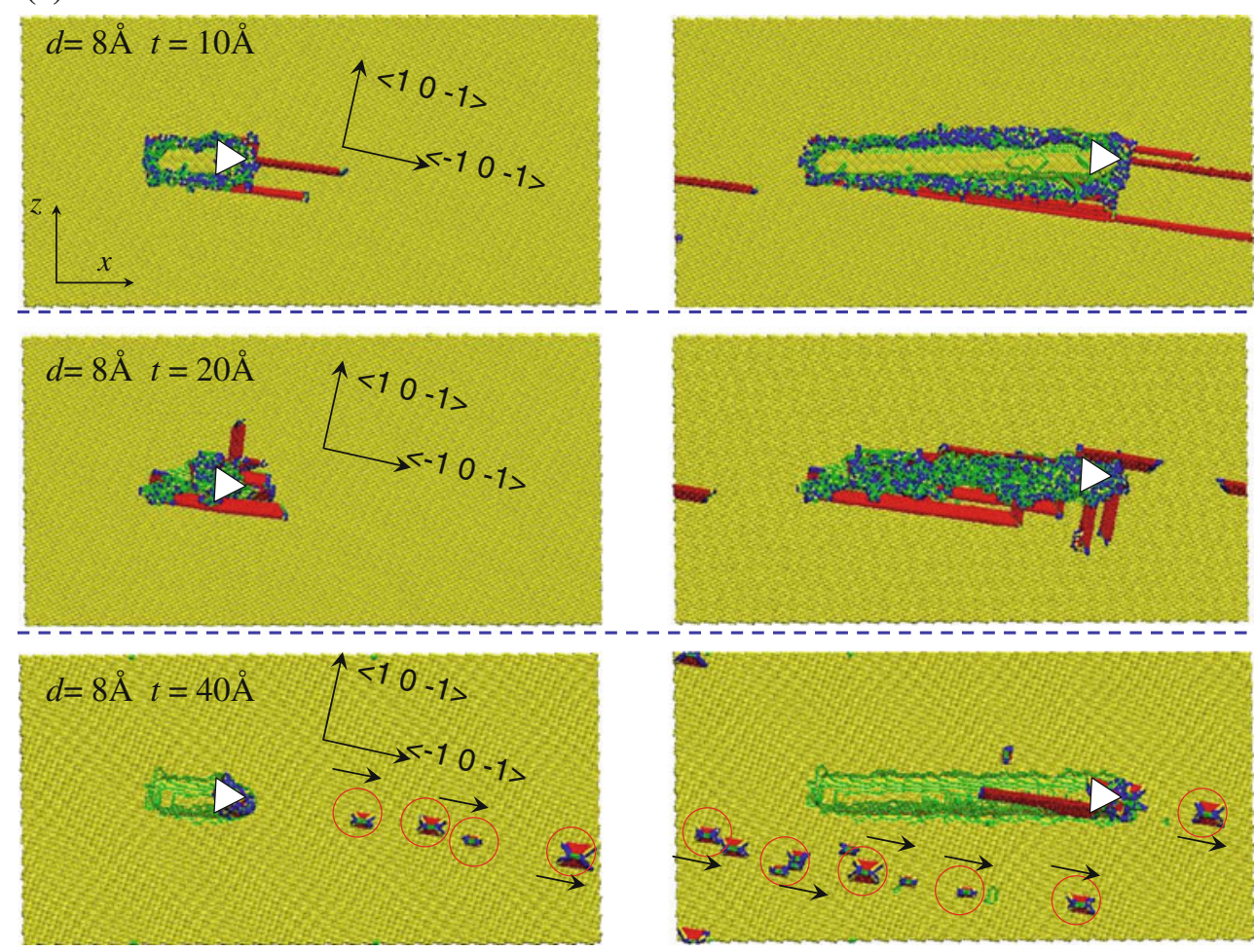

(b)
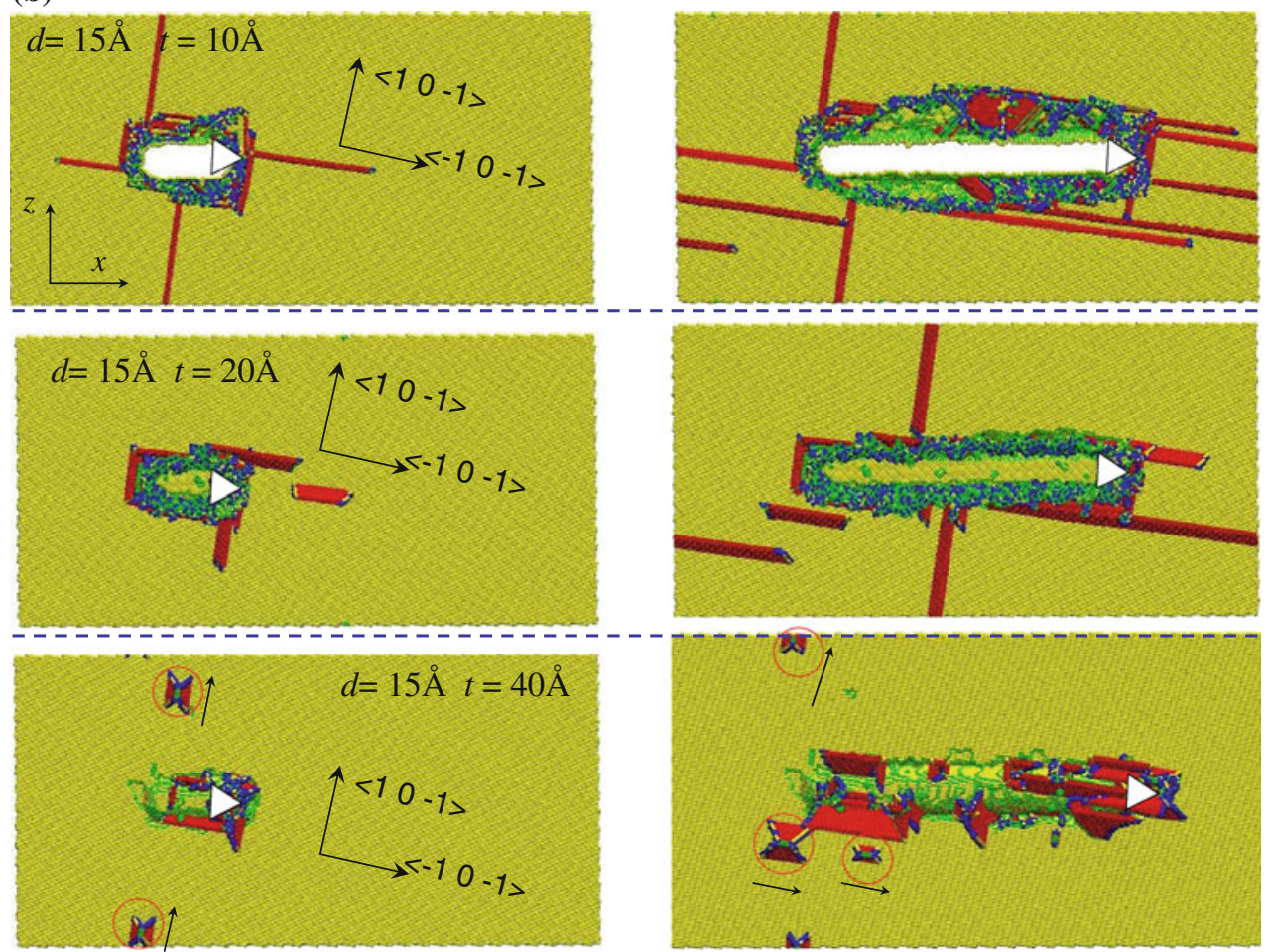

$F_{\text {stack }}<F_{\text {single }}<F_{\text {multi }}$

Thinner film leads to low friction since friction force is dominated by $\boldsymbol{F}_{\text {stack }}$, and the deeper scratch induces larger friction because friction is affected by $\boldsymbol{F}_{\text {multi }}$ at deep scratch.

Figure 9 shows the volume fraction diagrams during the scratch process. Figure. 9a shows the cutting view of the 
section which is perpendicular to the scratch direction. According to this sketch, we define the following variables:

$V_{\text {plas: }}$ the fraction of atoms which penetrates into the substrate after the scratch;

$V_{\text {plou }}$ : the fraction of atoms which ploughs on the two sides along the scratch track after the scratch;

$V_{\text {fil }}$ : the fraction of atoms which fills in the track after the scratch.

The volume fractions for both $\boldsymbol{d}=8$ and $15 \AA$ are shown in Fig. 9b, c, respectively, and indicate that the film interface plays a critical role in absorbing plastic deformations during the scratch. The $V_{\text {plas }}$ fraction decreases as
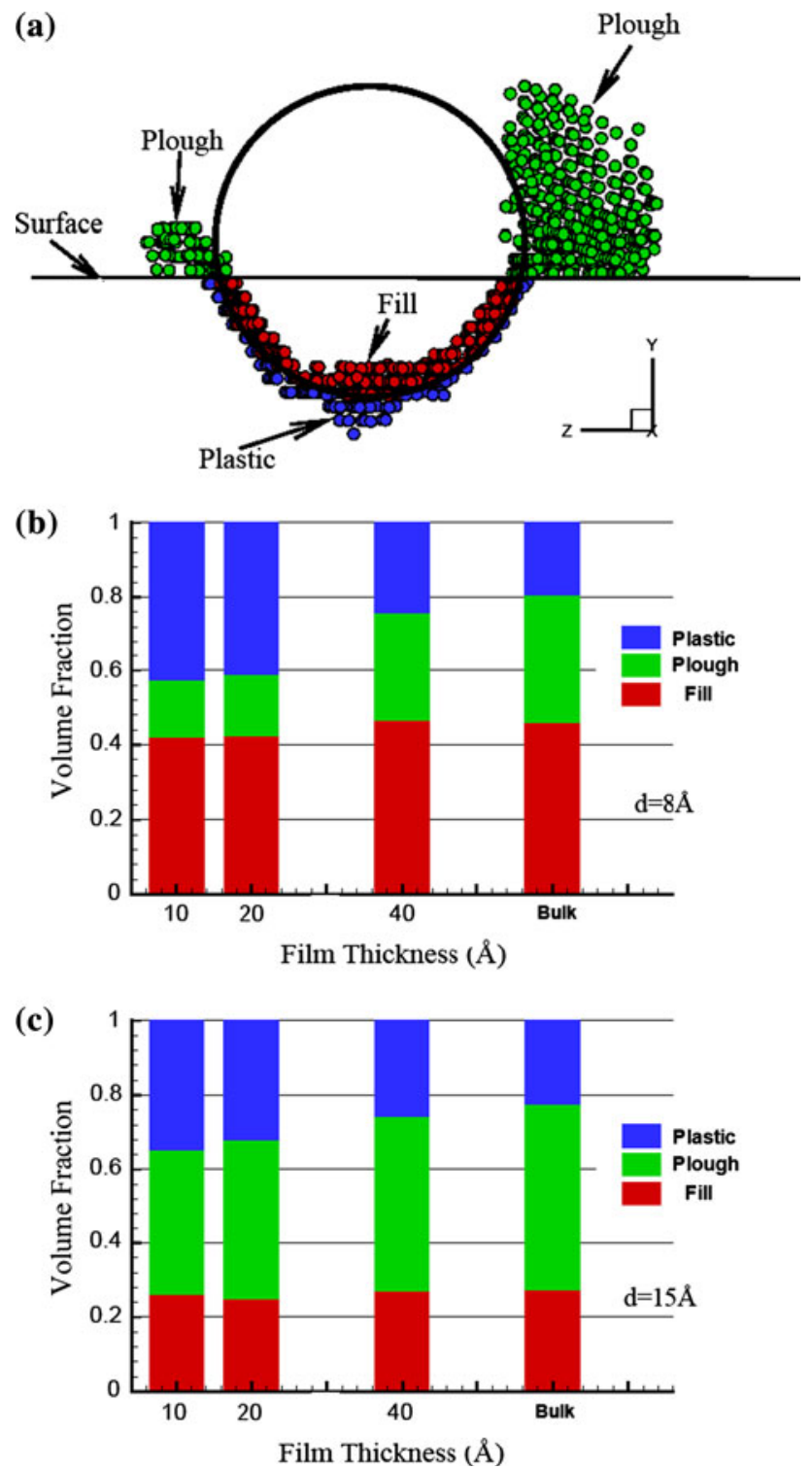

Fig. 9 Volume fractions during the scratch process. a Cutting view of the section which is perpendicular to the scratch direction, b volume fractions at scratch depth $\boldsymbol{d}=8 \AA$, c volume fractions at scratch depth $\boldsymbol{d}=15 \AA$ the film thickness $t$ increases. In the thinner film at both scratch depths, around $40 \%$ of the local deformation is released by the plastic part due to various contributions during contact, such as local interface slip, GB atom shuffling, and dislocation absorption and arresting of interfilm stacking fault at GB. $V_{\text {plou }}$ increases with increases in scratch depth, which in turn induces a larger contact area that causes a large friction force. This result verifies the same tribological trends observed in friction force variations in both Figs. 3 and 4. Compared with microscale scratch experiments on film [30], the conclusion drawn from our simulations is consistent with the finding of the former study, which is that spalling along the scratch track becomes more serious with increasing film thickness. If we check the atomistic configurations in Fig. 5, only piling up in front of the tip is observed in Fig. 5f-I and, as a consequence of piling up in front of the tip, a larger percentage of $V_{\text {plou }}$ forms after the tip moves forward. This finding is consistent with the variation of the $V_{\text {plou }}$ part in Fig. 9, with the increased larger contact area resulting in larger friction resistance.

\section{Comparison with Other Research}

Our results show friction coefficient $\mu$ ranging from $\sim 0.2$ to $\sim 0.6$ for various scratch depths. This range is similar with other MD results, although there are several other factors which might affect the friction ratio. Komanduri et al. performed nanoscratch on single crystal aluminum at extremely low depths, namely, $0-8 \AA$, to investigate atomic-scale friction [8, 23]. The friction coefficients at these depths are much higher $(>0.66)$ than our results because the sharp edge is used as the tip in their simulations. Mulliah et al. [31, 32] performed simulations by nanoscratch on a silver surface (100) and obtained a dynamic friction coefficient that ranged from 0.13 to 0.46 with increasing depth from 5 to $15 \AA$. These results are similar to our results shown in Fig. 4. The main differences between Mulliah et al.'s [31, 32] results and our results are that we obtained ultra-low friction at a depth of $5 \AA$ in some cases without taking into account the adhesive force. Gao et al. [33] studied the effect of indenter shape on the nanoscratch behavior of $\mathrm{Ni}$, obtaining a friction coefficient ranging from 0.31 to 0.43 for a round triangular tip at the scratch depth of $25 \AA$ with tip radius of $140 \AA$. From scratching experiments at nanoscale depth, the result of the friction coefficients for Ni film given by Islam et al. [34] ranged from 0.4084 to 0.4473 , which is quite similar to the value obtained in our simulations although the scratch velocity and temperature effect were not taken into account in our cases. 
Recent MD simulations for the contact of single asperity [35] indicate that subtle variations in the atomic structure on the contact surface substantially improve the mechanical properties, such as contact areas, adhesive forces, contact stiffness, as well as friction behavior. Although continuum mechanics models tend to fail at the nanoscale, Mo et al. [36] demonstrated that the failure is due to inaccurately defining the contact area in nanoscale, and they suggest that by defining the contact area as being proportional to the number of interacting atoms, the macroscopic linear relationship between friction force and contact area is able to be extended to the nanoscale. We plot the variation of friction force as a function of number of contact atoms, as shown in Fig. 10 where the rectangle symbols denote the nanoscratch results in bulk with several depths $(\boldsymbol{d}=8,10,12,15 \AA)$ and the triangle symbols denote the results in film. It can be seen that the bulk results agree well with the liner prediction by Mo [36]. In addition, because the effects of the interface on the thicker film $\boldsymbol{t}=40 \AA$ are small, these data also fall in with the linear fit. However, this linear prediction breaks down with decreasing film thickness. The number of contact atoms decreases with the introduction of the interface, and the decrease in friction force drops more rapidly, so that the film data fall below the linear prediction. This may provide a clue that decreases in the film thickness are able to improve the surface wear condition. From the wear experiment [37], specific wear rate is reduced dramatically with decreasing film thickness from 2,000 to $10 \mathrm{~nm}$, which may be related to the increasing effect of the interface with decreasing thickness. The interface makes the surface

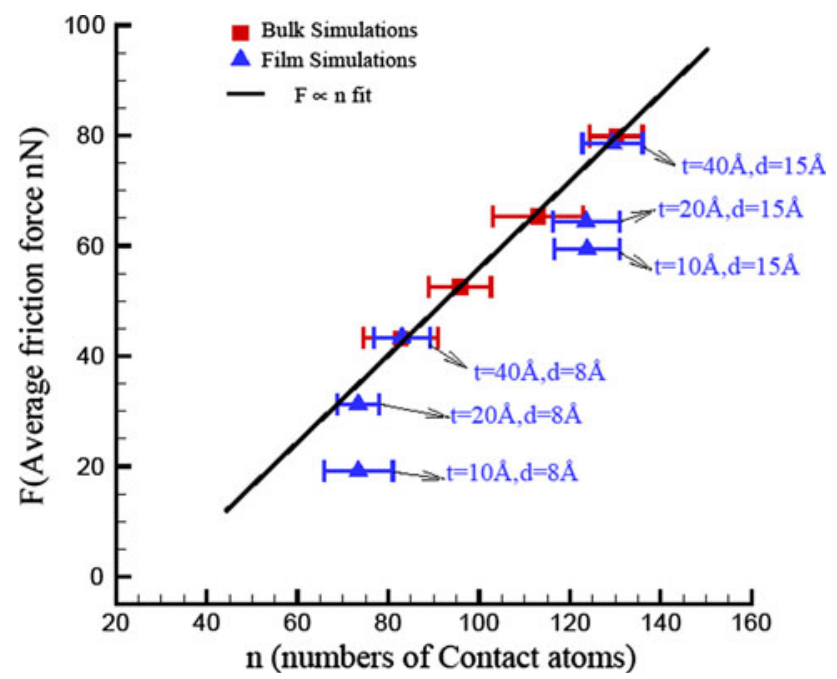

Fig. 10 Average friction force versus contact atom number. Solid line Linear dependence predicted by Mo et al. [36]. Error bars Standard deviation in these measurements at different scratch positions material have more resistance against scratching by mediating a large part of the deformation in front of the tip.

There are several factors which may affect the tribological properties of the surface. Müser [38] studied the kinetic friction dependence on temperature and scratch velocity by using the Prandtl-Tomlinson model. Also, as indicated by Zhu et al. [39, 40], a higher cutting velocity would result in a larger chip volume in front of the indenter, both cutting force as well as the normal force, and the temperature would affect the chip volume in front of the indenter. Sørensen et al. [41] reported that the friction force fluctuations were affected by the temperature and sliding speed, which were related with the excitation of phonons and energy dissipation. Fang et al. demonstrated
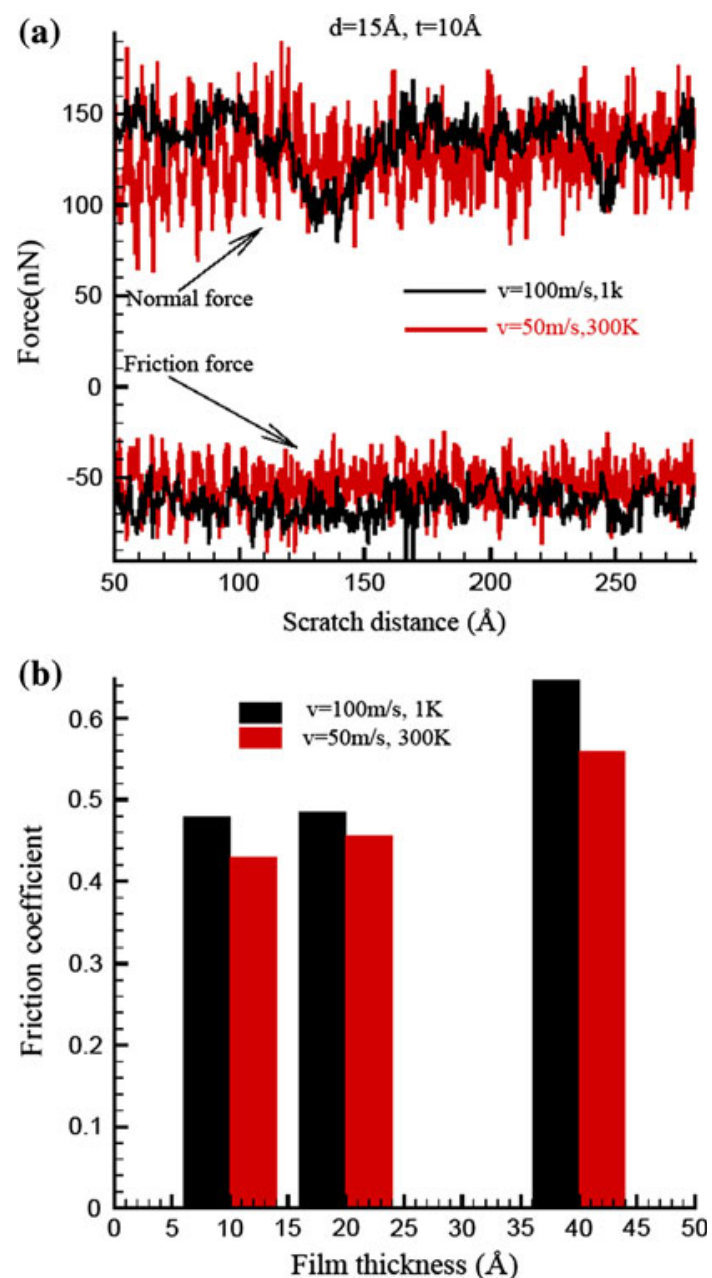

Fig. 11 Temperature and scratch velocity effects on the friction process. a Normal and friction force curves for the case of scratch depth $\boldsymbol{d}=15 \AA$ and film thickness $\boldsymbol{t}=10 \AA$ (black curves are the case of temperature $1 \mathrm{~K}$, scratch velocity $100 \mathrm{~m} / \mathrm{s}$; red curves are the case of temperature $300 \mathrm{~K}$ and scratch velocity $50 \mathrm{~m} / \mathrm{s}$ ). b Average friction coefficient as a function of film thickness at a scratch depth of $\boldsymbol{d}=15 \AA$ (red bars denote cases with scratch velocity $50 \mathrm{~m} / \mathrm{s}$ and temperature $300 \mathrm{~K}$; black bars denote cases with scratch velocity $100 \mathrm{~m} / \mathrm{s}$ and temperature $1 \mathrm{k}$ ) (Color figure online) 
that the thermostat and the indenter shape in MD simulations should be considered in a nanoscale contact model $[22,42]$. We also performed some simulations at a higher temperature and low scratch velocity for comparison, as shown in Fig. 11, which presents the friction behavior at scratch depth $\boldsymbol{d}=15 \AA$ at different temperatures and scratch velocities. It can be seen that the increase in temperature and decrease in scratch velocity induce a decrease of normal and friction force, as shown in Fig. 11a, and also cause a decrease in the average friction coefficient, as shown in Fig. 11b. However, Fig. 11 also clearly shows a similar interface effect on the change of friction coefficient as shown earlier-the friction coefficient is smaller at thinner film cases $t=10$ and $20 \AA$. In addition, studies show that surface structure, such as roughness [43] and self-affinity [44], can also plays a crucial role in affecting surface contact; these should also be considered in scratch process studies. The effect of temperature, contact velocity and interface geometry (indenter shape, surface structure) on the tribological properties of the thin film system will be further studied in our future work.

\section{Conclusion}

In the present study, three-dimensional MD simulations using the EAM potential were conducted to study the nonadhensive nanometric cutting process of $\mathrm{Ni}$ film. The effects of both film thickness and cutting depth on the nanoscale friction behavior have been investigated. Summaries are listed in Table 1, and the following conclusions have been drawn.

(1) Ultra-low friction is obtained at the fine cutting depth, which is around the displacement burst value. However, the low friction tends to break down as the film thickness decreases. Internal friction at GB results in the accumulation of local amorphous structure beneath the tip that may cause the change in friction behavior at the surface.

(2) Both scratch depth and film thickness affect nanoscale friction behaviors. Friction coefficient decreases with decreases in both the scratch depth and film thickness, with the exception of the case of very fine scratch depth $(\boldsymbol{d}=5 \AA)$.

(3) Internal friction at the GB mediates the large part of deformation generated during the scratch, and the interface slip between the film and the substrate releases the stress concentration around the indenter tip and further reduces both the material accumulation and friction coefficient.

(4) The interface affects the microstructure formation and evolution beneath the tip. The required force for forming trans-film stacking faults is smaller than that for nucleation of V-shape dislocations, and this is one of the reasons why the friction coefficient decreases with decreasing film thickness. Decreasing the film thickness can induce decreases in both ploughing and piling up, which may be an effective way of decreasing friction.

Acknowledgments This work was supported by the National Natural Science Foundation of China (Grants Nos. 10772096, 11021262, 10932011 and 91116003), and by the National Basic Research Program of China through 2012CB937500. The authors gratefully acknowledge useful discussions with Prof. F. P. Yuan from LNM at the Institute of Mechanics, Chinese Academy of Sciences.

\section{References}

1. Persson, B.N.J.: Sliding Friction: Physical Principles and Applications. Springer Verlag, Berlin (2000)

2. Bhushan, B.: Handbook of Micro/Nanotribology. CRC Press, Boca Raton (1999)

3. Greenwood, J.A., Wu, J.J.: Surface roughness and contact: an apology. Meccanica 36, 617-630 (2001)

4. Persson, B.N.J., Albohr, O., Tartaglino, U., Volokitin, A.I., Tosatti, E.: On the nature of surface roughness with application to contact mechanics, sealing, rubber friction and adhesion. J. Phys. Condens. Matter 17, R1 (2005)

5. Tomassone, M.S., Sokoloff, J.B., Widom, A., Krim, J.: Dominance of phonon friction for a xenon film on a silver (111) surface. Phys. Rev. Lett. 79, 4798-4801 (1997)

6. Baumgartl, J., Dietrich, J., Dobnikar, J., Bechinger, C., Von Gruenberg, H.H.: Phonon dispersion curves of two-dimensional colloidal crystals: the wavelength-dependence of friction. Soft Matter 4, 2199-2206 (2008)

7. Juaristi, J.I., Alducin, M., Muino, R.D., Busnengo, H.F., Salin, A.: Role of electron-hole pair excitations in the dissociative adsorption of diatomic molecules on metal surfaces. Phys. Rev. Lett. 100, 116102 (2008)

8. Komanduri, R., Chandrasekaran, N., Raff, L.M.: Molecular dynamics simulation of atomic-scale friction. Phys. Rev. B. 61, 14007 (2000)

9. Fang, T.H., Liu, C.H., Shen, S.T., Prior, S.D., Ji, L.W., Wu, J.H.: Nanoscratch behavior of multi-layered films using molecular dynamics. Appl. Phys. A. Mater. Sci. Process. 90, 753-758 (2008)

10. Cho, M.H., Kim, S.J., Lim, D.S., Jang, H.: Atomic scale stick-slip caused by dislocation nucleation and propagation during scratching of a $\mathrm{Cu}$ substrate with a nanoindenter: a molecular dynamics simulation. Wear 259, 1392-1399 (2005)

11. Hardy, W.B., Doubleday, I.: Boundary lubrication. The paraffin series. Proc. R. Soc. Lond. Ser. A. 100, 550 (1922)

12. Madou, M.J.: Fundamentals of Microfabrication: The Science of Miniaturization. CRC Press, Boca Raton (2002)

13. Misra, A., Hirth, J.P., Hoagland, R.G.: Length-scale-dependent deformation mechanisms in incoherent metallic multilayered composites. Acta Mater. 53, 4817-4824 (2005)

14. Dayal, P., Savvides, N., Hoffman, M.: Characterisation of nanolayered aluminium/palladium thin films using nanoindentation. Thin Solid Films 517, 3698-3703 (2009)

15. Medyanik, S.N., Shao, S.: Strengthening effects of coherent interfaces in nanoscale metallic bilayers. Comput. Mater. Sci. 45, 1129-1133 (2009) 
16. Kang, B.C., Kim, H.Y., Kwon, O.Y., Hong, S.H.: Bilayer thickness effects on nanoindentation behavior of $\mathrm{Ag} / \mathrm{Ni}$ multilayers. Scripta Mater. 57, 703-706 (2007)

17. Saraev, D., Miller, R.E.: Atomistic simulation of nanoindentation into copper multilayers. Model. Simul. Mater. Sci. Eng. 13, 1089 (2005)

18. Cheng, D., Yan, Z.J., Yan, L.: Misfit dislocation network in $\mathrm{Cu} /$ Ni multilayers and its behaviors during scratching. Thin Solid Films 515, 3698-3703 (2007)

19. Foiles, S.M., Baskes, M.I., Daw, M.S.: Embedded-atom-method functions for the fcc metals $\mathrm{Cu}, \mathrm{Ag}, \mathrm{Au}, \mathrm{Ni}, \mathrm{Pd}, \mathrm{Pt}$, and their alloys. Phys. Rev. B. 33, 7983 (1986)

20. Kelchner, C.L., Plimpton, S.J., Hamilton, J.C.: Dislocation nucleation and defect structure during surface indentation. Phys. Rev. B. 58, 11085 (1998)

21. Plimpton, S.: Fast parallel algorithms for short-range molecular dynamics. J. Comp. Phys. 117, 1-19 (1995)

22. Fang, T.H., Chang, W.Y., Huang, J.J.: Dynamic characteristics of nanoindentation using atomistic simulation. Acta Mater. 57, 3341-3348 (2009)

23. Komanduri, R., Chandrasekaran, N., Raff, L.M.: MD simulation of indentation and scratching of single crystal aluminum. Wear 240, 113-143 (2000)

24. Nosé, S.: A unified formulation of the constant temperature molecular dynamics methods. J. Chem. Phys. 81, 511 (1984)

25. Choi, Y., Van Vliet, K.J., Li, J., Suresh, S.: Size effects on the onset of plastic deformation during nanoindentation of thin films and patterned lines. J. Appl. Phys. 94, 6050 (2003)

26. Kluge, M.D., Wolf, D., Lutsko, J.F., Phillpot, S.R.: Formalism for the calculation of local elastic constants at grain boundaries by means of atomistic simulation. J. Appl. Phys. 67, 2370-2379 (1990)

27. Lilleodden, E.T., Zimmerman, J.A., Foiles, S.M., Nix, W.D.: Atomistic simulations of elastic deformation and dislocation nucleation during nanoindentation. J. Mech. Phys. Solids 51, 901-920 (2003)

28. Gosvami, N.N., Filleter, T., Egberts, P., Bennewitz, R.: Microscopic friction studies on metal surfaces. Tribol. Lett. 39, 19-24 (2010)

29. Humphrey, W., Dalke, A., Schulten, K.: VMD: visual molecular dynamics. J. Mol. Graph. 14, 33-38 (1996)
30. Qi, J., Chan, C.Y., Bello, I., Lee, C.S., Lee, S.T., Luo, J.B., et al.: Film thickness effects on mechanical and tribological properties of nitrogenated diamond-like carbon films. Surf. Coat. Technol. 145, 38-43 (2001)

31. Mulliah, D., Kenny, S.D., Smith, R.: Modeling of stick-slip phenomena using molecular dynamics. Phys. Rev. B. 69, 205407 (2004)

32. Mulliah, D., Kenny, S.D., Smith, R., Sanz-Navarro, C.F.: Molecular dynamic simulations of nanoscratching of silver (100). Nanotechnology 15, 243 (2004)

33. Gao, Y., Lu, C., Huynh, N.N., Michal, G., Zhu, H.T., Tieu, A.K.: Molecular dynamics simulation of effect of indenter shape on nanoscratch of Ni. Wear 267, 1998-2002 (2009)

34. Islam, S., Ibrahim, R.N.: Mechanism of abrasive wear in nanomachining. Tribol. Lett. 42, 275-284 (2011)

35. Luan, B., Robbins, M.O.: The breakdown of continuum models for mechanical contacts. Nature 435, 929-932 (2005)

36. Mo, Y., Turner, K.T., Szlufarska, I.: Friction laws at the nanoscale. Nature 457, 1116-1119 (2009)

37. Wilson, G.M., Sullivan, J.L.: An investigation into the effect of film thickness on nanowear with amorphous carbon-based coatings. Wear 266, 1039-1043 (2009)

38. Müser, M.H.: Velocity dependence of kinetic friction in the Prandtl-Tomlinson model. Phys. Rev. B. 84, 125419 (2011)

39. Zhu, P., Hu, Y., Ma, T., Wang, H.: Study of AFM-based nanometric cutting process using molecular dynamics. Appl. Surf. Sci. 256, 7160-7165 (2010)

40. Zhu, P., Hu, Y., Ma, T., Wang, H.: Molecular dynamics study on friction due to ploughing and adhesion in nanometric scratching process. Tribol. Lett. 41, 41-46 (2011)

41. Sørensen, M.R., Jacobsen, K.W., Stoltze, P.: Simulations of atomic-scale sliding friction. Phys. Rev. B. 53, 2101 (1996)

42. Chang, W.Y., Fang, T.H., Lin, S.J., Huang, J.J.: Nanoindentation response of nickel surface using molecular dynamics simulation. Mol. Simul. 36, 815-822 (2010)

43. Persson, B.N.J.: Elastoplastic contact between randomly rough surfaces. Phys. Rev. Lett. 87, 116101 (2001)

44. Campaná, C., Müser, M.H.: Contact mechanics of real vs. randomly rough surfaces: a Green's function molecular dynamics study. Europhys. Lett. 77, 38005 (2007) 